\title{
The Use of Robotics in Stroke Rehabilitation
}

\author{
Marie-Helene Milot \\ Centre de recherche sur le vieillissement; Université de Sherbrooke \\ 1036 Belvédère sud, Sherbrooke, Canada \\ marie-helene.milot@usherbrooke.ca
}

\section{Extended Abstract}

In Canada, strokes affect about 62000 people each year, which is equivalent to a stroke occurring every 9 minutes [1]. Following a stroke, many survivors have difficulty undergoing voluntary movement in their affected limb. Both the spatial components (i.e. direction of movement) and the temporal components of movement (i.e. reaction time) are affected, compromising the functional performance of the individuals with hemiparesis. In order to minimize this negative impact, robotic training is an innovative technique that is recommended more and more as a rehabilitative strategy post stroke [2]. Indeed, several studies using various robots and training protocols have found notable improvements such as in motor recovery and social participation [3] following stroke. Yet, no clear consensus prevails in the literature regarding the ideal robotic training parameters and robotic design to ensure optimal functional recovery of stroke survivors.

The objective of this talk is to provide ideas on what could be the optimal training parameters (e.g. type of training, feedback) as well as robotic design (e.g. available range of motion) to warrant treatment gains post stroke. Thus, the results of my past and current works in rehabilitation robotics of stroke survivors will be presented. For examples, from a previous study with the arm exoqueleton BONES [4], it was shown that a sophisticated multijoint robotic training was not a critical variable of improvement in motor function of the trained limb. It was also suggested that robots, designed to allow training of the full range of motion of the trained limb, could allow a better transfer of training gains to improvement in performance of daily task by stroke survivors. Finally, using a simple one-degree-of-freedom robot, TEO, the impact of two different robotic training interventions--haptic guidance and error amplification, were evaluated in order to improve movement timing post stroke. Interestingly, the results showed that the side of the stroke lesion is important to consider in the design of robotic training since it influenced how stroke survivors responded to the robotic training interventions [5]. Based on these results, my current research program is focusing on the design of a simple end-effector robot that allows training the upper limb in its full range of motion. This robot will be tested in an upcoming study aimed at improving affected upper limb movement using haptic guidance and error amplification robotic training interventions.

The scope of this talk will be to generate discussions on what should be the next step in the design of robots as well as robotic training parameters to ensure the clinical applicability of this therapeutic tool for a neurologically impaired population.

\section{References}

[1] Heart and Stroke Foundation (2016, February 24). Stroke Report 2015 [Online]. Available: $\mathrm{http} / / / \mathrm{www} \cdot$ heartandstroke.com/site/apps/nlnet/content2.aspx?c=ikIQLcMWJtE\&b=7498307\&ct=14715105\&utm_ca mpaign=offline\&utm_source=strokereport\&utm_medium=vanity.

[2] N. Norouzi-Gheidari, et al, "Effects of robot-assisted therapy on stroke rehabilitation in upper limbs: systematic review and meta-analysis of the literature," J Rehabil Res Dev, vol. 49, pp. 479-96, 2012.

[3] A. C. Lo, et al, "Robot-assisted therapy for long-term upper-limb impairment after stroke," N Engl J Med, vol. 362, pp. 1772-83, 2010.

[4] M. H. Milot, et al, "A crossover pilot study evaluating the functional outcomes of two different types of robotic movement training in chronic stroke survivors using the arm exoskeleton BONES," J Neuroeng Rehabil, vol. 10, p. 112, 2013.

[5] A. E. Bouchard, et al, "Guiding movement or increasing movement error in chronic stroke survivors: which robotic intervention is best to boost the learning of a timing task (submitted manuscript)," Disabil Rehabil, 2016. 